\title{
Exceptional lability of a genomic complex in rice and its close relatives revealed by interspecific and intraspecific comparison and population analysis
}


Jianxin $\mathrm{Ma}^{1 *}$, Scott A Jackson ${ }^{1 *}$

\begin{abstract}
Background: Extensive DNA rearrangement of genic colinearity, as revealed by comparison of orthologous genomic regions, has been shown to be a general concept describing evolutionary dynamics of plant genomes. However, the nature, timing, lineages and adaptation of local genomic rearrangement in closely related species (e. g., within a genus) and haplotype variation of genomic rearrangement within populations have not been well documented.

Results: We previously identified a hotspot for genic rearrangement and transposon accumulation in the Orp region of Asian rice (Oryza sativa, AA) by comparison with its orthologous region in sorghum. Here, we report the comparative analysis of this region with its orthologous regions in the wild progenitor species $(O$. nivara, AA) of Asian rice and African rice (O. glaberrima) using the BB genome Oryza species (O. punctata) as an outgroup, and investigation of transposon insertion sites and a segmental inversion event in the AA genomes at the population level. We found that Orp region was primarily and recently expanded in the Asian rice species $O$. sativa and $O$. nivara. LTR-retrotransposons shared by the three AA-genomic regions have been fixed in all the 94 varieties that represent different populations of the AA-genome species/subspecies, indicating their adaptive role in genome differentiation. However, LTR-retrotransposons unique to either $O$. nivara or $O$. sativa regions exhibited dramatic haplotype variation regarding their presence or absence between or within populations/subpopulations.
\end{abstract}

Conclusions: The LTR-retrotransposon insertion hotspot in the Orp region was formed recently, independently and concurrently in different AA-genome species, and that the genic rearrangements detected in different species appear to be differentially triggered by transposable elements. This region is located near the end of the short arm of chromosome 8 and contains a high proportion of LTR-retrotransposons similar to observed in the centromeric region of this same chromosome, and thus may represent a genomic region that has recently switched from euchromatic to heterochromatic states. The haplotype variation of LTR-retrotransposon insertions within this region reveals substantial admixture among various subpopulations as established by molecular markers at the whole genome level, and can be used to develop retrotransposon junction markers for simple and rapid classification of O. sativa germplasm.

\footnotetext{
*Correspondence: maj@purdue.edu; sjackson@purdue.edu

'Department of Agronomy, Purdue University, West Lafayette, IN 47907, USA

Full list of author information is available at the end of the article
} 


\section{Background}

Comparative genetic mapping and comparison of orthologous genomic sequences of grasses, such as rice, maize, sorghum, barley, wheat, and millet have demonstrated extensive genomic colinearity among species that radiated from common ancestors $\sim 10-60$ million years ago $[1,2]$. Although numerous and different levels of genomic rearrangements, including gene movement, and loss or creation of new genes were uncovered in some grass lineages [3-5], gene content has been shown to be highly conserved between species. For instance, all genes, including singlecopy ones, absent in the genomic region surrounding the orange pericarp (Orp) gene of rice, in contrast to its orthologous regions of sorghum and maize, were found elsewhere in the rice genome and even in Arabidopsis $[3,6]$. Comparison of homoeologous segments of maize revealed exceptionally high-level of loss of one of the homoeologous gene pairs [3,5-7], which appears to be a general phenomenon in the evolution of any polyploid organism toward a diploid genomic state. These dynamic processes of gene duplication and deletion may explain why rice and Arabidopsis share a similar set of genes, although their genomes have undergone separate paleopolyploidy and/or segmental duplication events during their $\sim 120$ million year independent evolution [8].

In contrast to genes, intergenic spaces were found to be less or not conserved between grasses, such as maize and sorghum. Intergenic sequences are generally composed of transposable elements or transposable element fragments, primarily long terminal repeat (LTR)-retrotransposons, and other unknown DNA components. Given that most structurally detectable LTR-retrotransposons were amplified within last a few million years $[9,10]$, it is not surprising that substantial differences in intergenic regions have been found between subspecies of rice [11], or even between inbreds of maize $[12,13]$. On the other hand, LTR-retrotransposons can be partially or completely deleted from the host genomes within very short evolutionary timeframes. For example, it was estimated that $\sim 200 \mathrm{Mb}$ of LTR-retrotransposon DNA was removed from the rice genome by unequal homologous recombination and illegitimate recombination within the past 5 million years $[9,10]$, although neither amplification nor removal of LTR-retrotransposons seems to be absolutely gradual processes [14]. In addition to the gain and loss of transposable elements, intergenic sequences generally diverge more rapidly than genic sequences by nucleotide substitution $[10,15]$. These dynamic processes have led to the scarcity of conserved intergenic sequences, even between moderately diverged grass lineages such as maize and sorghum $[6,16]$.

Comparison of closely related species, subspecies, and/ or different haplotypes or ecotypes is a promising approach to investigate more recent evolutionary events.
A comparative sequence analysis of $\sim 1.1 \mathrm{Mb}$ orthologous regions of two subspecies of rice, indica and japonica, revealed more than $2 \%$ and $6 \%$ growth of two respective genomes over the past half million years, primarily by amplification of LTR-retrotransposons [10]. Wang and Dooner presented a comprehensive comparison of seven inbred lines of maize, demonstrating the remarkable haplotype variation of the $b z$ genomic regions caused predominantly by insertion of LTR-retrotransposons, helitrons, DNA transposons and other new repetitive components [17]. However, the dynamic variation of transposable elements, their potential interplay with genic rearrangement, and their roles for genomic selection and diversity remain to be investigated, particularly, at the population level.

The high-quality genomic sequence of rice [18] and genomic resources (e.g., BAC libraries, BAC end sequences, BAC-based physical maps) generated by the ongoing Oryza map alignment project (OMAP) [19] provide an unprecedented opportunity for research community to study the evolution of plant genomes within a genus. To date, three genomic (Adh1, MOC1, and $H d 1$ ) regions of multiple Oryza species have been investigated [20-22]. Because the Oryza species included in OMAP span evolutionary scales from $<1$ million years to $\sim 15$ million years, as indicated by their phylogeny [23], comparisons of multiple Oryza species in these regions have uncovered some specific evolutionary events in specific lineages during the radiation of the Oryza species. However, all three regions are gene-rich and repeat-poor, therefore little is known about how transposable elements have affected the instability of the Oryza genomes during their speciation and diversification.

A hotspot of transposable element accumulation that harbors a few truncated and duplicated gene fragments was previously described between two gene clusters of the Orp region of rice (O. sativa ssp. japonica). This hotspot is located near the end of the short arm of chromosome 8 (from 1757 to $1997 \mathrm{~kb}$, rice Pseudomolecule 4.0), and contains a high proportion of LTR-retrotransposons, similar to that observed in the centromeric region (Cen8) of this same chromosome [6,24], but it is absent in the corresponding regions of sorghum and maize [6]. To track the evolutionary history of the formation of this hotspot and the spectrum of genic rearrangements involved, we identified its orthologous regions from AA-, BB-, EE-, and FF-genome Oryza species by searching the O. sativa Orp region against BAC end sequences (BESs) generated by OMAP. In particular, we sequenced two overlapping BAC clones from $O$. nivara (AA), one of the proposed wild progenitors of Asian rice (O. sativa), one BAC clone from O. glaberrima (AA), the cultivated rice species domesticated in African, and one BAC clone from O. punctata (BB). In 
addition, we investigated the haplotype variation of LTR-retrotransposon insertions and an inversion of a genomic segment within the hotspot. We present here the comparative genomic analysis of these orthologous regions and haplotype variation mediated by LTR-retrotransposons, thereby depicting the nature, timing, rate and specificity of DNA changes observed in these regions during the speciation and diversification of these closely related Oryza species.

\section{Results and Discussion}

Size variation of the Orp orthologous regions across Oryza species reflects recent genomic expansion

To select the genomic segments that are orthologous to the O. sativa Orp regions in other Oryza species, we searched a set of BAC end sequences (BESs) derived from the O. nivara, O. glaberrima, O. punctata, O. australiensis, and O. brachyantha genomes (OMAP, http:// www.omap.org, Figure 1A). Individual BAC clones with two BESs anchored to the unique sequences of the $O$. sativa Orp region and/or its flanking regions in opposite orientations were considered to be orthologous segments. One or two overlapping BAC clones from each species that maximally cover the hotspot of insertions of transposable elements in the Oryza Orp region [6] were chosen for sequencing and/or further analysis. BAC clones OR_BBa0014L06 from O. nivara, two overlapping clones OG_BBa0075G14 and OGBBa0001L21 from $O$. glaberrima, and clone OP_Ba0008J05 from O. punctata were completely sequenced. These sequenced genomic segments are $214 \mathrm{~kb}, 190 \mathrm{~kb}$, and $192 \mathrm{~kb}$ in O. nivara, O. glaberrima, and O. punctata, respectively, corresponding to $202 \mathrm{~kb}, 309 \mathrm{~kb}$, and $339 \mathrm{~kb}$ of the orthologous regions in $O$. sativa (Table 1 ). Thus, a $12-\mathrm{kb}$ expansion of the O. nivara region and $119-\mathrm{kb}$ and $147-$ $\mathrm{kb}$ contractions of the O. glaberrima and O. punctata regions relative to the corresponding orthologous regions in $O$. sativa were revealed. In addition, $212-\mathrm{kb}$ and $192-\mathrm{kb}$ contractions of the $O$. australiensis and $O$. brachyantha regions relative to the corresponding $O$. sativa orthologous regions (Table 1 ) were suggested by fingerprinting of BAC clones OA_ABa0108F22 and OB_Ba0050L03 (OMAP, http://www.omap.org) that define the orthologous Orp regions in O. australiensis and $O$. brachyantha, respectively (Figure $1 \mathrm{~B}$ ). The relative contraction of the Orp region in sorghum in contrast to the Orp region in O. sativa is $175 \mathrm{~kb}$ (Table 1 ), and no transposable elements were identified in the sorghum region [6]. These observations, together with the evolutionary relationship of these species as illustrated in Figure 1A, suggest that the Orp region was primarily and recently expanded in the Asian rice species $O$. sativa and $O$. nivara [i.e., after the divergence of Asian and African rice approximately 1.2 million years ago
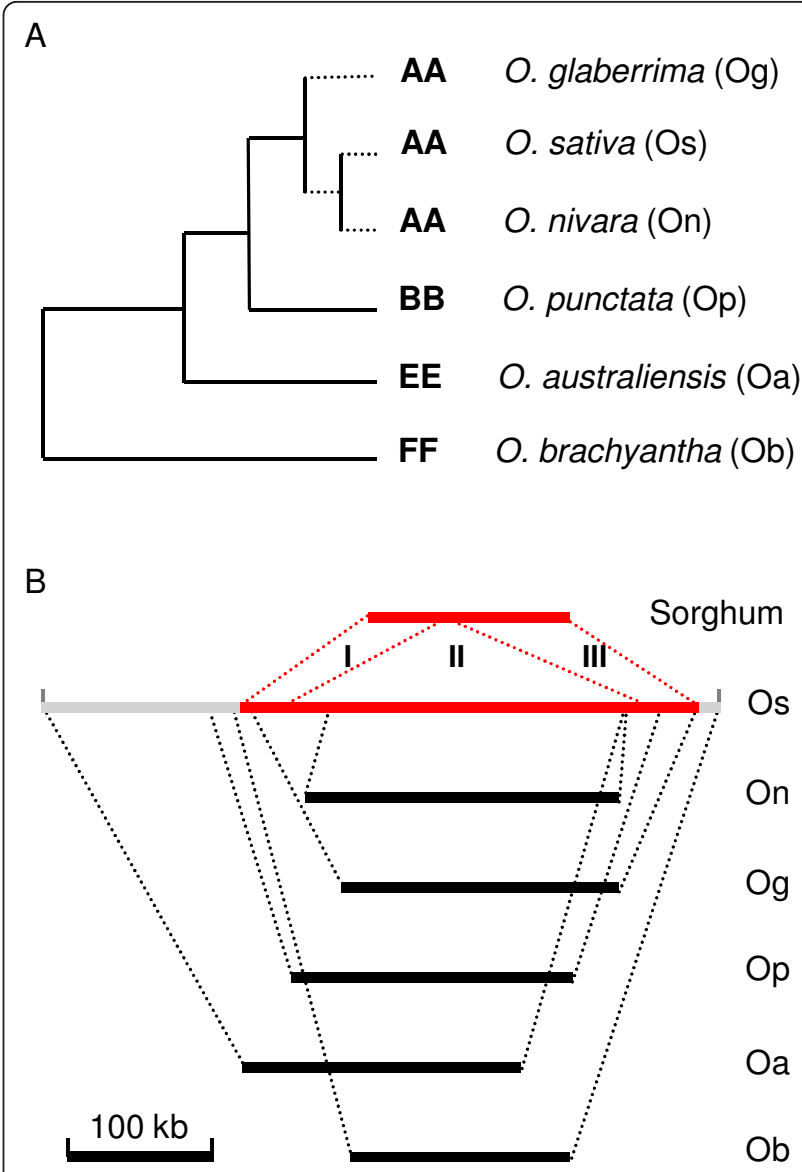

Figure 1 Size variation of the orthologous Orp regions of Oryza species. (A) Phylogeny of the Oryza species [adapted from [23]]. (B) Contraction/expansion of the Orp regions in O. nivara, $O$. glaberrima, $\mathrm{O}$. punctata, $\mathrm{O}$. australiensis, and $\mathrm{O}$. brachyantha relative to the orthologous region in $O$. sativa. Dotted red lines connecting the $O$. sativa and sorghum regions mark two gene clusters interrupted by a hotspot of transposon insertions and genic rearrangements in O. sativa as previously described by Ma et al. 2005. Dotted dark lines mark the boundaries of the orthologous regions defined by anchoring BESs from other Oryza species to the O. sativa sequence of the Orp region.

[25]]. Because this study aimed to decipher the nature and timing of recent genic rearrangements and gain and loss of LTR-retrotransposon in the Orp regions of the AA genome species, the orthologous BAC clones from O. australiensis and O. brachyantha were not further investigated.

\section{Sequence organization and comparison of the orthologous regions}

Analysis of the Orp orthologous regions in O. sativa and sorghum was previously performed [6]. Using these same criteria, we annotated the orthologous regions from O. nivara, O. glaberrima, and O. punctata. Sequence organizations of the five orthologous regions 
Table 1 Size variation of the orthologous Orp regions of Oryza investigated

\begin{tabular}{|c|c|c|c|c|c|c|c|}
\hline \multirow[b]{2}{*}{ Species $^{a}$} & \multirow{2}{*}{$\begin{array}{c}\text { Genome } \\
\text { designation }\end{array}$} & \multirow{2}{*}{$\begin{array}{l}\text { Genome } \\
\text { size }^{\mathrm{b}} \\
(\mathrm{Mb})\end{array}$} & \multirow[b]{2}{*}{ BAC or contig } & \multirow{2}{*}{$\begin{array}{c}\begin{array}{c}\text { Size of BAC or } \\
\text { contig }\end{array} \\
(k b)^{c}\end{array}$} & \multirow{2}{*}{$\begin{array}{c}\text { Size of corresponding } \\
\text { region in } \\
\text { O. sativa }(k b)^{d}\end{array}$} & \multicolumn{2}{|c|}{$\begin{array}{c}\text { Size variation relative } \\
\text { to } 0 \text {. sativa }(\mathrm{kb})\end{array}$} \\
\hline & & & & & & Contraction & Expansion \\
\hline O. nivara & $\mathrm{AA}$ & 448 & OR_BBa0014L06 & 214 & 202 & & 12 \\
\hline O. glaberrima & $\mathrm{AA}$ & 354 & $\begin{array}{l}\text { OG_BBa0075G14/ } \\
\text { OG_BBa0001L21 }\end{array}$ & 190 & 309 & 119 & \\
\hline O. punctata & BB & 423 & OP_Ba0008J05 & 192 & 339 & 147 & \\
\hline O. australiensis & $\mathrm{EE}$ & 960 & OA_Aba0108F22 & 190 & 401 & 212 & \\
\hline O. brachyantha & $\mathrm{FF}$ & 338 & OB_Ba0050L03 & 150 & 342 & 192 & \\
\hline Sorghum & $\mathrm{HH}$ & 735 & SB18C08 & $138^{e}$ & $313^{e}$ & 175 & \\
\hline
\end{tabular}

a Oryza species refers to Wing et al., 2005 and sorghum refers to Ma et al., 2005.

${ }^{\mathrm{b}}$ Refers to Bennett and Leitch, 2003.

${ }^{\mathrm{C}}$ Estimated by gel electrophoreses of restriction enzyme-digested fragments or sequencing.

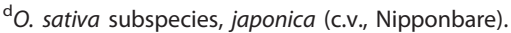

'The corresponding region comparable.

are illustrated in Figure 2 and detailed in Additional file 1, Table S1.

The 214-kb O. nivara region is comprised of 21 LTRretrotransposons, 3 DNA transposons, 3 Helitrons, and 6 genes/pseudogenes. LTR-retrotransposons alone make up $121 \mathrm{~kb}$ of DNA sequence, accounting for $56.6 \%$ of the region. Although the $O$. nivara region is only $12 \mathrm{~kb}$ larger than its corresponding orthologous region in
O. sativa, it was found that 12 LTR-retrotransposons (74 kb of DNA) in the former and 9 LTR-retrotransposons (55 kb of DNA) in the latter, were not shared in the two regions. Each of these unshared elements is flanked by 5 -bp target site duplication in its host region with a single copy of the 5-bp "target site" in the other orthologous region, suggesting that these elements inserted into the current positions after the divergence

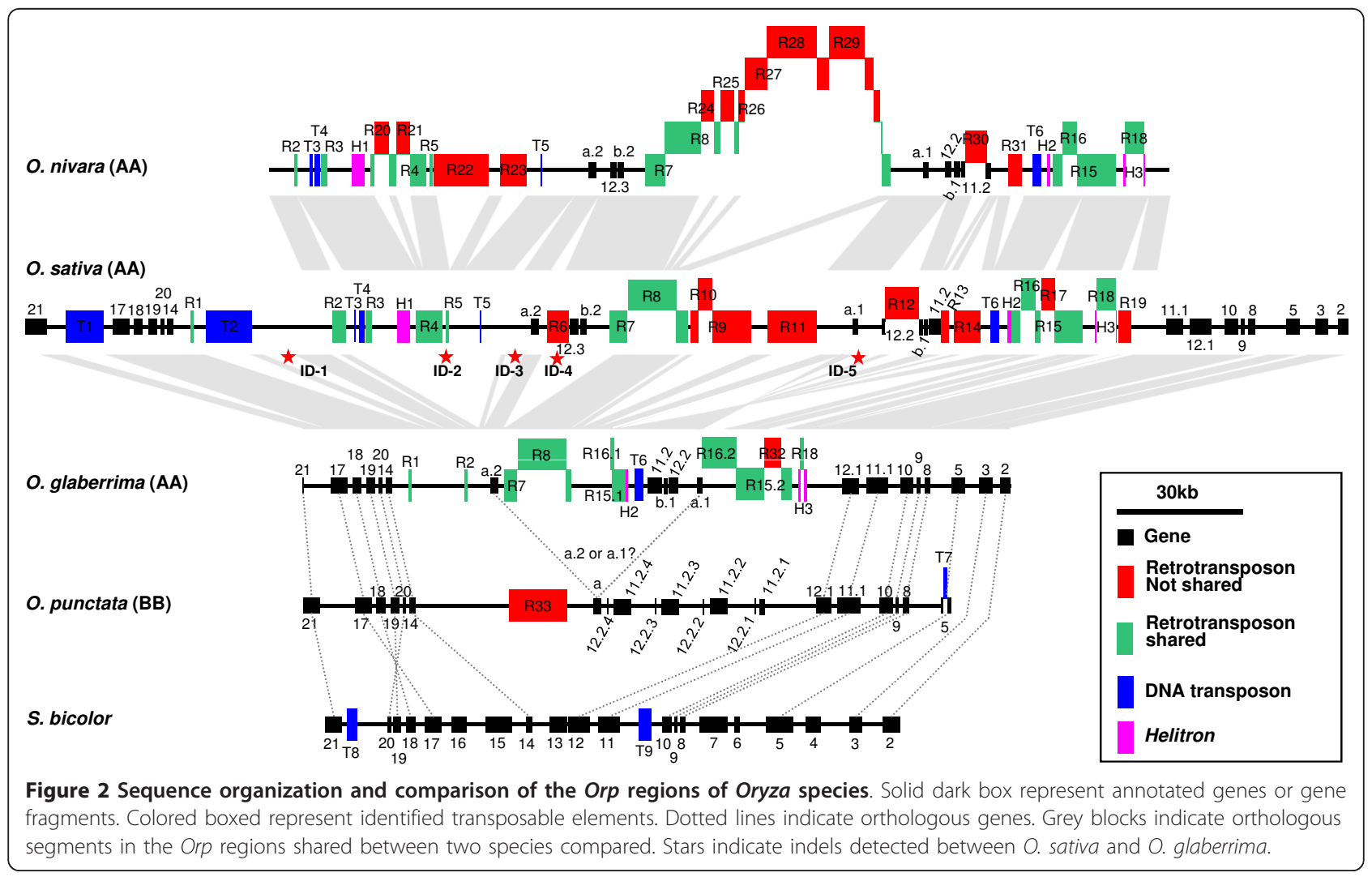


of the two regions from their ancestral form. By contrast, all of the 3 DNA transposons and 3 Helitrons are shared in the two regions, suggesting that they were integrated before the divergence of the two regions. Overall, these two regions exhibit perfect colinearity in gene order and orientation, although gene 11.2 in $O$. nivara and gene 12.2 in O. sativa each had a LTR-retrotransposon insertion (Figure 2).

The 190-kb O. glaberrima region is comprised of 7 LTR-retrotransposons, 1 DNA transposon and 2 Helitrons. Nine out of these 10 transposable elements are shared by $O$. glaberrima and $O$. sativa, indicating that the O. glaberrima region did not expand substantially by the amplification of LTR-retrotransposons after its divergence from the $O$. sativa lineage. In addition to indels (insertions/deletions) generated by insertions of 2 DNA transposons (T1 and T2) and 8 LTR-retrotransposons (R9, R10, R11, R12, R13, R14, R17, and R18), and by the formation of a solo LTR (R2) through unequal recombination [26] in O. glaberrima, 5 relatively large indels (> $3 \mathrm{~kb}$ ), ID-1, ID-2, ID-3, ID-4 and ID-5 (Figure 2 ) were observed between the two regions. Among these 5 indels, ID-2 is the largest ( $37 \mathrm{~kb})$ and harbors 3 DNA transposons, 1 Helitron, and 3 LTR-retrotransposons present in O. sativa. Genes 12.3 and b.2 involved in ID4. were present in $O$. sativa but absent in O. glaberrima. ID- 5 appears to be a $(4.3 \mathrm{~kb})$ deletion that led to partial truncation of gene a1 and removal of a DNA transposon $(4.3 \mathrm{~kb})$ fragment in O. glaberrima. This deletion flanks the inverted segment in the O. glaberrima region that harbors genes 11.2, b1 and 12.2. The other breakpoint for this inversion is located within LTR-retrotransposon R16 (belonging to family Osr $14[27,28]$ ) and as a result R16 was separated into two fragments (R16.1 and R16.2). Interestingly, a deletion of $\sim 4.3 \mathrm{~kb}$ internal sequence of R16 at this breakpoint was deduced by comparing R16.1 and R16.2 with typical intact elements of the Osr14 family $[27,28]$. It is unclear whether the inversion led to the two flanking deletions or the latter caused the former. The O. glaberrima and O. sativa regions show overall colinearity except for the genic rearrangements described above.

The 148-kb O. punctata region has a single LTR-retrotransposon (R33), which is not shared by the three Agenome species. Based on the divergence of two LTRs of R33, it was estimated that this element was integrated into the region $\sim 0.038$ mya. This region shares perfect colinearity at the two gene clusters (i.e., genes $5,8,9$, $10,11,12$, and genes $14,20,19,18,17,21$ ) with the Agenome species, with the exception of a recent quaduplication of a segment containing two gene fragments (genes 11.2 and 12.2), which resulted in a substantial size increase of the interval between the two gene clusters in O. punctata.
The nature and history of genic rearrangements

Most duplicated genes interspersed in the intervals of the two highly conserved gene clusters are pseudogenes or gene fragments, in which the protein-coding sequences cannot be accurately predicted. Thus, the gene duplication events observed in this study could not be dated based on protein-coding sequences. To illuminate the history of the duplication events, we performed phylogenetic analysis of the duplicated genes within and across species using their genomic sequences (Figure 3). As shown in Figure 3A, gene 11.1 and gene 11.2 from the three species, O. sativa, O. glaberrima, and O. punctata, were grouped into two distinct branches (i.e., gene 11.1 branch and gene 11.2 branch), and the phylogeny reflected by either branch is consistent with the evolutionary relationship among the three species [23]. These data suggest that gene 11.1 and gene 11.2 were duplicated before the divergence of $\mathrm{AA}$ and $\mathrm{BB}$ genome species from a common ancestor. Gene 12.2 (12.2.1, 12.2.2, 12.2.3 and 12.2.4) in O. punctata was largely truncated, and thus excluded in the phylogenetic analysis. Gene 12.1 in the three species shows a similar phylogenetic pattern (Figure 3B) as revealed by gene 11.1 and 11.2,

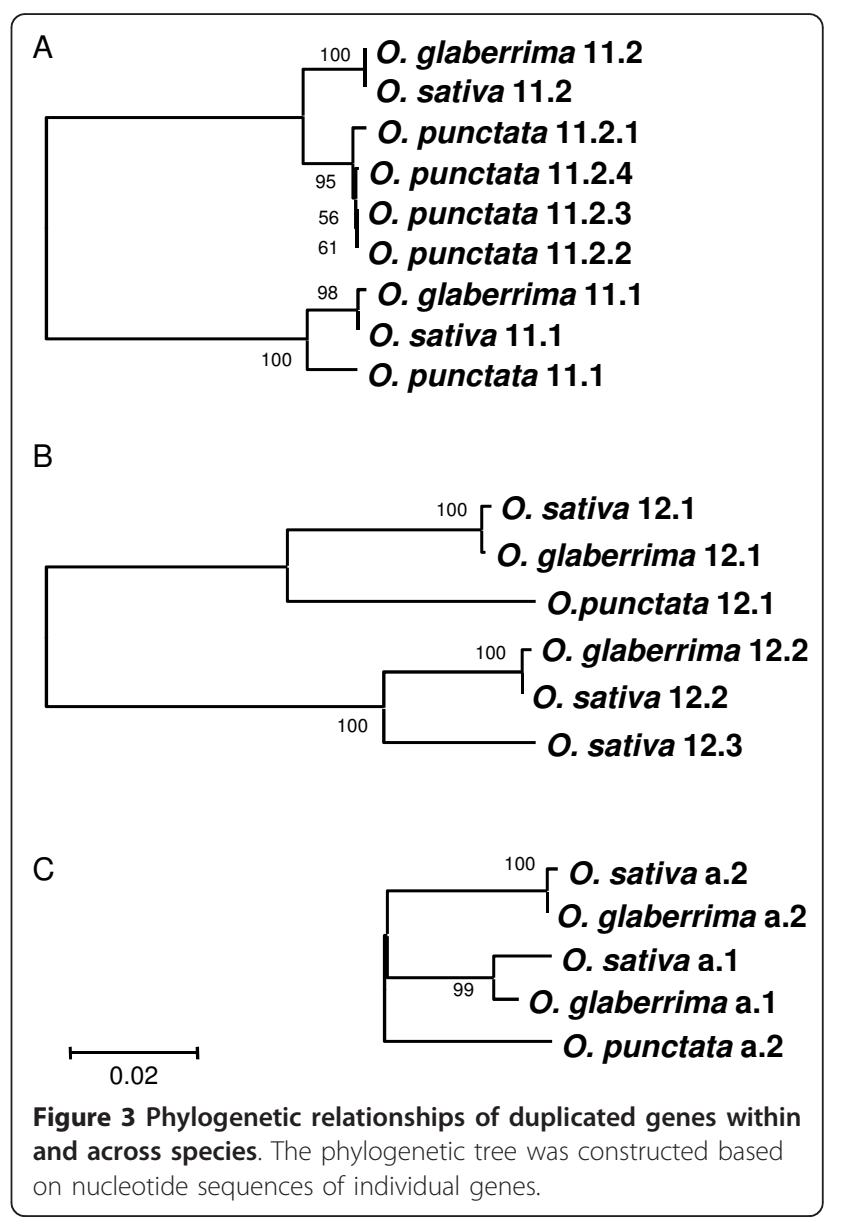


suggesting that the duplication of gene 12.1 and 12.2 also occurred before the divergence of the AA and $\mathrm{BB}$ genomes. The genetic distances between genes 11.1 and 11.2 and between genes 12.1 and 12.2 differ (Figure 3A and $3 \mathrm{~B}$ ), but the genes 11.1 and 12.1 and genes 11.2 and 12.2 were found to be arranged in the same order and orientation in the duplicated fragments. Thus, it is most likely that the duplication of both genes was caused by a single event.

Genes 12.2 and 12.3 in $O$. sativa grouped in the same branch, distinct to the branch of gene 12.1, suggesting that the duplication of 12.2 and 12.3 occurred after the first duplication event that predates the divergence of the $\mathrm{AA}$ and $\mathrm{BB}$ genomes. Gene b.1 is present in the AA genome species, but absent in the $\mathrm{BB}$ genome and sorghum. If the conserved segments containing genes 11.1 and 12.1 are ancestral copies of genes 11 and 12, the insertion of gene b (b.1 or b.2) must have occurred after the first duplication event. Because the three genes in each of the two gene clusters (a.2, 12.3 and b.2 cluster, and a.1, 12.2, and b.1 cluster) are arranged in the same order and orientation, it is likely that these three genes were duplicated by a single event before the divergence of the Asian and African AA genomes. The levels of sequence divergences between genes 12.2 and 12.3 and between genes a. 1 and a.2 in $O$. sativa are similar (Figure 3B and 3C), reinforcing this conclusion. Assuming this deduction is correct, the absence of genes 12.3 and b.2 in O. glaberrima must be the outcome of deletion(s) at ID-4 site (Figure 2).

Phylogenetic analysis revealed that gene a (a.1 or a.2) in O. punctata is nearly equally distinct to genes a.1 and a.2 in either AA genome species (Figure $3 \mathrm{C}$ ), suggesting that the duplication of the gene a (i.e., a.1 and a.2) occurred near the split of the AA and BB genome species. Thus, the orthologous copy/copies of genes a between the AA and BB genomes cannot be deduced based on their sequence similarities. Phylogenetic analysis indicates that the four recently amplified copies of gene 11.2 in O. punctata are orthologous to gene 11.2 in $O$. sativa and $O$. nivara (Figure $3 \mathrm{~A}$ ). In comparison with the proposed two orthologous regions in the AA genomes, genes 12.3 , b.2, b.1 and a.1 were absent in the $\mathrm{BB}$ genome (Figure 2).

According to the analyses above, we propose two evolutionary scenarios regarding genic arrangements and rearrangements in the Oryza Orp regions. The first scenario, as illustrated in Figure 4A, proposes that the initial copy of gene a (i.e., a.1) inserted before the divergence of the $\mathrm{AA}$ and $\mathrm{BB}$ genome, and the initial copy of gene $b$ (i.e., b.1) inserted only in the AA species after the AA and BB species divergence. The duplication event was followed by the duplication of the gene cluster (a.1, 12.2 and b.1) that generated genes a.2, 12.3, and b. 2 in the AA species. Based on this hypothesis, the absence of genes 12.3, b.2, b.1 and a.1 in the BB genome can be explained solely by "gain" of these genes in the Orp regions of the AA species. Of course, it is also possible that the insertions of initial copies of genes $a$ and $b$ and the subsequent duplication of the gene cluster (a.1, 12.2 and b.1) occurred before the divergence of the AA and $\mathrm{BB}$ species (Figure $4 \mathrm{~B}$ ). In this scenario case, the absence of genes seen in the $B B$ genome could be explained by multiple gene deletion events, which is less parsimonious than the first scenario.

Regardless, our data revealed unusual structural instability in the Oryza Orp regions, including recent and rapid accumulation of LTR-retrotransposons and recent genic rearrangements. These genomic changes took place within an originally gene-rich euchromatic chromosome arm, reflecting a general plasticity of the Oryza genomes under the umbrella of local genic colinearity. Given that the structural variation of genomic regions can substantially affect chromatin states [29], frequencies of local recombination [13], and the expression/functionality of genes within or flanking the regions [30], the genomic plasticity revealed in this region, and probably many other genomic regions, as a general pattern, may have played a significant role, as proposed by Ginzburg et al. [31], in the processes of Oryza genome speciation.

\section{Population analysis of haplotype variation of LTR- retrotransposon insertions and segmental inversion in the AA species}

Previous investigation of the $b z$ genomic region in seven different maize inbred lines revealed remarkable variation in the maize genome, structure mediated by transposable elements $[17,32]$. Similar to the $b z$ region, the Orp regions of the three Oryza AA genomes show a high level of polymorphisms of LTR-retrotransposon insertions (Figure 2). To further track whether a particular LTR-retrotransposon is present at high frequencies or fixed within a species/subspecies at population levels, we investigated the presence or absence of a set of LTRretrotransposons identified in the Orp regions by PCR amplification of transposon insertion junctions in $95 \mathrm{AA}$ genome varieties, following a protocol previously described by Devos et al. [1] (see details in Materials and Methods). These 95 varieties, including $46 O$. sativa, 20 O. nivara, 24 O. rufipogon and 4 O. glaberrima and $1 O$. barthii accessions (Additional file 2, Table S2) were chosen based on their geographic distribution and genetic diversity estimated by SSR and SNP markers [33-35]. The results of PCR analysis are illustrated in Figure 5 and Additional file 3, Figure S1. The primers designed for PCR analysis are listed in Additional file 4, Table S3. 


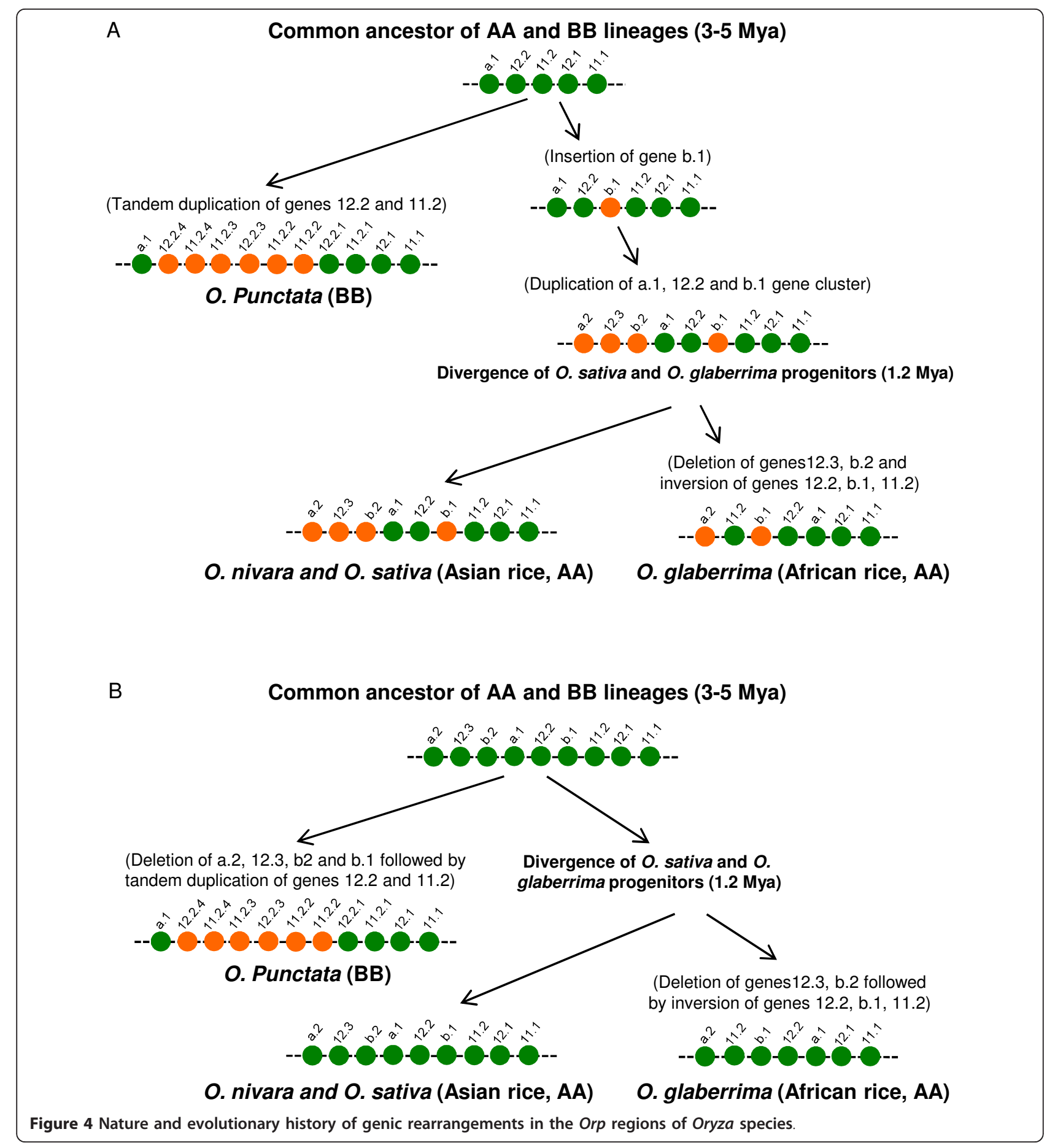

R7 and R15, two representative LTR-retrotransposons shared by the three AA genomic regions, were detected in all the 94 AA genome varieties (Figure 5). In other words, these two insertions were fixed during the evolution and divergence of the AA genome species. It was estimated that these two elements were inserted approximately 2.1 and 1.2 million years ago. In general, LTR-retrotransposons tend to accumulate in low recombination heterochromatic regions where selection is expected to be less efficient in removing them [36]. Thus, the fixation of the insertion of these two elements inserted before the divergence of African and Asian rice lineages in the "originally" gene-rich Orp region with high rate of recombination [28] would suggest that these elements may have played or be playing an adaptive role. 


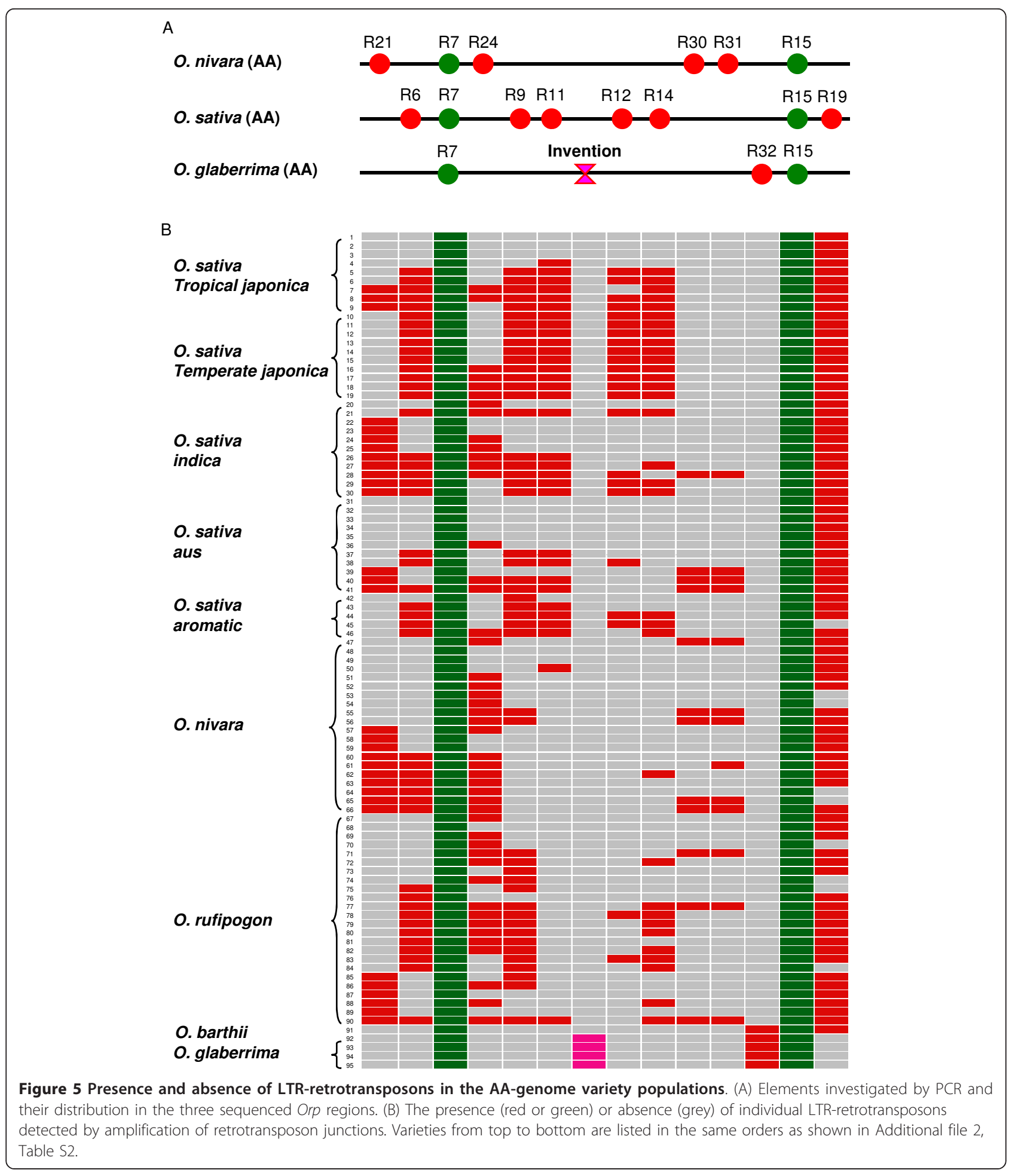

We also investigated 11 LTR-retrotransposons that are not shared among the three sequenced Orp regions, including 6 (R6, R9, R11, R12, R14, and R19) unique to the O. sativa region, 4 ( $\mathrm{R} 21, \mathrm{R} 24, \mathrm{R} 30$, and $\mathrm{R} 31)$ unique to the O. nivara region, and 1 (R32) unique to the O. glaberrima region. These elements were relatively young (Additional file 1, Table S1) with an average age of 0.15 million years. R32 was found in the four O. glaberrima varieties, but absent in all Asian AA varieties, suggesting that this element was inserted into the African AA lineage after 
its divergence from the Asian AA lineage. R19 was found in 47 out of the 48 O. sativa varieties, being absent from a single aromatic/GroupV accession, and it was detected in 16 out of the $20 \mathrm{O}$. nivara varieties and 20 out of $24 \mathrm{O}$. rufipogon varieties. Interestingly, R6, R9, R11, R12 and R14, which distinguished Nipponbare from $O$. nivara, were fixed in the 10 temperate japonica varieties, while R21, R30 and R31, which were present in the sequenced $O$. nivara $\mathrm{BAC}$, were completely absent from the temperate japonica subpopulation (Figure 5). A similar pattern was seen for R21, R30 and R31 (all absent), and for R6, R9 and R11 (all present) in the aromatic/GroupV accessions, which are known to be closely related to temperate japonica at the genetic level [37]. On the other hand, the tropical japonica varieties, as a group, were more similar to the indica and aus varieties across this region, with varying frequencies of LTRs matching those found in Nipponbare and/or $O$. nivara. Interestingly, the indica variety, 9311, which is known to have japonica parentage, shares a regional haplotye with temperate japonica, as do the two Indonesian tropical japonica varieties, Gotak Gatik and Trembese, while most of the others in this group carry the $O$. nivara alleles at R9, R112, R12 and R14. The indica subpopulation is almost fixed for the O. nivara allele at R21, and is highly variable across all other markers. The aus subpopulation is distinguished from the other $O$. sativa groups by a higher frequency of accessions carrying R30 and R31 and the complete absence of accessions carrying both R12 and R14. In these ways, aus more closely resembles $O$. nivara than other O. sativa varieties across this region. These observations are consistent with previous studies showing an intense genetic bottleneck in temperate japonica but greater variation in the indica and aus subgroups [33,38] and substantial admixture among the various subpopulations, most notably in tropical japonica [35].

Based on the presence/absence of all these LTR-retrotransposons, "phylogenetic relationships" of these varieties were analyzed. As shown in Additional file 5, Figure S2, the analysis clearly separated the African species, O. glaberrima and $O$. barthii (the proposed wild progenitor of O. glaberrima) from the Asian species, and the indica and aus subpopulations clustered at one end of the graph along with most of the O. nivara and some of the O. rufipopgon accessions, well separated from the majority of aromatic/Group V, temperate and tropical japonica varieties that clustered with a different set of O. rufipogon accessions. This analysis further identifies admixed tropical japonica, indica and aus varieties that cluster with several O. nivara and O. rufipogon accessions in the middle of the graph. Several indica varieties are seen clustering with the japonica group, reflecting the greater genetic variation and mixed parentage of many indica varieties, as previously noted for c.v. 9311. Thus, this graphical display reflects the taxonomy of these species and subspecies as established by SNP and/or SSR analyses, providing an interesting window on a highly variable region of the rice genome [33-35].

Several hypotheses can explain the observed lability of LTR-retrotransposons. 1) Since the regions are highly instable and plastic, there may be a high level of lineage sorting going on in present-day populations derived from a very diverse set of ancestral haplotypes. Each descendant population may inherit a large subset of the ancestral haplotypes, which continue to segregate in the descendants. In theory, over evolutionary time they should sort out such that each group has its own distinct haplotype/haplotypes that are more closely related to each other than to haplotypes from other species. However, because of the relatively short time that has elapsed for the speciation of the Asian AA genomes, the haplotypes of LTR-retrotransposons remain largely unsorted. 2) The lability can be explained by intra-specific and inter-specific introgression, which may have occurred during speciation of these genomes $[35,39,40]$. 3) Balancing selection for recent LTR-retrotransposon insertions may contribute to the high level of insertion polymorphisms, although adaptive selection and/or genetic bottlenecks affecting the two relatively old elements, R7 and R15, was suggested. Further investigation of a larger collection of wild and cultivated germplasm and more LTR-retrotransposon insertions at a larger genomic scale would help to reveal the dynamics of retention and/or removal of LTR-retrotransposons and their contributions to genomic diversity and speciation.

The inverted segment harboring genes 11.2, b1 and 12.2 in the sequenced O. glaberrima region (Figure 2) was detected in all other O. glaberrima accessions analyzed by PCR approach, but absent in the $O$. barthii accession and all Asian AA-genome Oryza species/subspecies (Figure 5 and Additional file 3, Figure S1). This suggests that the inversion occurred in African rice after its divergence from Asian rice. Because only a single $O$. barthii accession was included in this analysis, it remains unclear whether the inversion took place before or after the domestication of O.glaberrima from O. barthii.

The Orp region is located near the end of the short arm of rice chromosome 8 , but harbors a high proportion of LTR-retrotransposons similar to that observed in the centromeric region of this same chromosome. Thus, it is likely that the region has recently switched from euchromatic to heterochromatic states.

\section{Conclusions}

Our data indicate that the Orp genomic complex in rice cultivars and their wild progenitors have been recently, independently and concurrently formed from a gene-rich region by differential insertion of LTR-retrotransposons 
and genic rearrangement, and that the overall haplotype variation of LTR-retrotransposon insertions in this region echoes to the admixture pattern of genomic diversity and introgression of AA-genome populations/subpopulations revealed by genome-wide SSR and SNP genotyping, thus highlighting the evolutionary roles of LTR-retrotransposons in plant speciation and diversification. Genomewide profiling of LTR-retrotransposon insertions among the AA-genome cultivars at larger population levels would enhance our understanding of the evolutionary processes and dynamics of the rice genomes.

\section{Methods}

\section{Identification of BAC clones}

The entire Orp region of O. sativa and it flanking 150$\mathrm{kb}$ sequences from both ends of the region were searched against the BESs of other Oryza species generated by OMAP [19]. Single BAC clones meeting the following criteria: 1) at least one unique end; 2) both ends aligned to the extended Orp region of O. sativa in forward/reverse pairs; and 3) both ends spanning 100 to $500 \mathrm{~kb}$ of $O$. sativa sequences, were considered to be the orthologous segments from the respective Oryza species. As the major objective of this study was to target the genomic space corresponding to the hotspot of the transposable element accumulation and genic rearrangement in $O$. sativa, we only selected and analyzed a minimum number of clones from Oryza species that maximally cover the target region, as shown in Figure 1.

\section{BAC Sequencing}

Shotgun libraries for selected BAC clones were constructed as described previously [41]. Subclones were sequenced from both directions using ABI PRISM BigDye Terminator Chemistry (Applied BioSystems, Foster, $\mathrm{CA}$ ) and run on an ABI3730 capillary sequencer. BAC clones were sequenced at approximately 8-10-fold redundancies, and then were assembled and finished to standard high quality sequences (PHASE III) by primer walking [6]. The assemblies of sequenced BAC clones were confirmed by restriction map analysis similar to the method described by Dubcovsky et al. [41].

\section{Sequence annotation}

Putative gene models were predicted using the FGENESH program with the monocot training set (http:// www.softberry.com), and were further investigated to determine whether they are actually genes following described previously criteria [6]. Truncated gene fragments were identified by sequence homology comparison using BLAST2 [42], DOTTER [43] and CROSS_MATCH (http://www.phrap.org). LTR-retrotransposons were identified and classified as described previously [9]. DNA transposon fragments were identified by homology

searches against the TIGR plant repeat database [44], GenBank non-redundant protein database, and pack_MULE database [45]. Helitrons were identified using a perl script described previously [46].

\section{Dating of segmental duplication and retrotransposon insertions}

The alignments of homologous nucleotide sequences were generated by using ClustalX [47]. The dates of segmental duplication and amplification of LTR-retrotransposons were estimated as described previously [10]. The phylogenetic trees of duplicated genes were constructed based on pair-wise comparison of nucleotide sequences using the Kimura two-parameter method provided by MEGA4 program [48]. The Neighbor-Joining tree based on the presence/absence of LTR-retrotransposon insertions were obtained using MEGA4.

The genomic sequences generated in this study has been deposited in GeneBank (Nos. HM999006HM999008).

\section{Additional material}

Additional file 1: Sequence annotation of different BACs in this study. ${ }^{*} 1605848$ to 2060871 region of O. sativa subspecies japonica (c.v., Nipponbare) gemone sequece (Pseudomolecule 4.0). ** Intact-tsd, intact element flanked by target site duplication; solo-tsd; solo LTR flanked by target site duplication; intact-notsd, intact element without target site duplication; solo-notsd; solo LTR without target site duplication; \&, internal sequence of LTR-retrotransposon; s, truncated fragment.

Additional file 2: The AA-genome Oryza varieties used in this study These 95 varieties, including 46 O. sativa, 20 O. nivara, 24 O. rufipogon, 4 O. glaberrima, and 10 . barthii accessions, were chosen based on their geographic distribution and genetic diversity.

Additional file 3: PCR verification of a segmental inversion present in $O$. glaberrima, but absent in $O$. sativa and $O$. nivara varieties. (A) Schematic primer design for amplification of inversion boundaries. (B) PCR products of primer pairs F1/R1 (panel b) and F2/R2 (panel c), and Waxy genes (panel a, control). Primers are shown in Additional file 4. Varieties are numbered according to their orders in Additional file 2.

Additional file 4: Primers used in this study. Primers used to check the polymorphisms of LTR-retrotransposon insertions and the inversion in different species/subspecies.

Additional file 5: Neighbor-Joining "Phylogeny" of the AA-genome varieties constructed using MEGA4 based on the presence/absence of a set of LTR-retrotransposons in individual varieties as illustrated in Figure 5. Varieties are numbered according to their orders shown in Figure 5 and Additional file 2.

\section{Acknowledgements}

We thank Jennifer Kimball of Cornell University for sending DNA extractions of the 95 Oryza accessions used in this analysis, Michael Purugganan for constructive comments and discussion on this work, and Teri Rambo and Paul Parker for technical support. This study was supported by National Science Foundation grant nos. IOS_0424833, DBI 0321678, DBI 0501877, and DBI 0606461) and the Purdue University College of Agriculture.

\section{Author details}

${ }^{1}$ Department of Agronomy, Purdue University, West Lafayette, IN 47907, USA. ${ }^{2}$ Arizona Genomics Institute, The University of Arizona, Tucson, AZ 85721, 
USA. ${ }^{3}$ Genomics Core Facility, Purdue University, West Lafayette, IN 47907 USA. ${ }^{4}$ Department of Plant breeding and Genetics, Cornell University, NY 14853, USA. ${ }^{5}$ Department of Biological Sciences, Purdue University, West Lafayette, IN 47907, USA.

\section{Authors' contributions}

$\mathrm{ZT}, \mathrm{YaY}, \mathrm{FL}, \mathrm{YeY}$, and PJS carried out the BAC sequencing and haplotype variation experiments. ZT, PJS, RAW, SRM, JM and SAJ participated in data analysis, ZT, SRM and JM contributed to the manuscript preparation. All authors read and approved the final manuscript.

\section{Received: 18 November 2010 Accepted: 8 March 2011}

Published: 8 March 2011

\section{References}

1. Devos KM, Ma J, Pontaroli AC, Pratt LH, Bennetzen JL: Analysis and mapping of randomly chosen bacterial artificial chromosome clones from hexaploid bread wheat. Proc Natl Acad Sci USA 2005, 102:19243-19248.

2. Messing J, Bennetzen JL: Grass genome structure and evolution. Genome Dyn 2008, 4:41-56.

3. Lai J, Ma J, Swigonova Z, Ramakrishna W, Linton E, Llaca V, Tanyolac B, Park YJ, Jeong OY, Bennetzen $J$, et al: Gene loss and movement in the maize genome. Genome Res 2004, 14:1924-1931.

4. Lai J, Li Y, Messing J, Dooner HK: Gene movement by Helitron transposons contributes to the haplotype variability of maize. Proc Nat Acad Sci USA 2005, 102:9068-9073.

5. Bruggmann R, Bharti AK, Gundlach H, Lai J, Young S, Pontaroli AC, Wei F, Haberer G, Fuks G, Du C, et al: Uneven chromosome contraction and expansion in the maize genome. Genome Res 2006, 16:1241-1251.

6. Ma J, SanMiguel P, Lai J, Messing J, Bennetzen JL: DNA rearrangement in orthologous orp regions of the maize, rice and sorghum genomes. Genetics 2005, 170:1209-1220.

7. Langham RJ, Walsh J, Dunn M, Ko C, Goff SA, Freeling M: Genomic duplication, fractionation and the origin of regulatory novelty. Genetics 2004, 166:935-945

8. Bennetzen JL, Coleman C, Liu R, Ma J, Ramakrishna W: Consistent overestimation of gene number in complex plant genomes. Curr Opin Plant Biol 2004, 7:732-736.

9. Ma J, Devos KM, Bennetzen JL: Analyses of LTR-retrotransposon structures reveal recent and rapid genomic DNA loss in rice. Genome Res 2004, 14:860-869.

10. Ma J, Bennetzen JL: Rapid recent growth and divergence of rice nuclear genomes. Proc Natl Acad Sci USA 2004, 101:12404-12410.

11. Han $B, X u e Y$ : Genome-wide intraspecific DNA-sequence variations in rice. Curr Opin Plant Biol 2003, 6:134-138.

12. Fu H, Dooner HK: Intraspecific violation of genetic colinearity and its implications in maize. Proc Natl Acad Sci USA 2002, 99:9573-9578.

13. Dooner HK, He L: Maize genome structure variation: interplay between retrotransposon polymorphisms and genic recombination. Plant Cell 2008, 20:249-258.

14. Bennetzen JL, Ma J, Devos KM: Mechanisms of recent genome size variation in flowering plants. Ann Bot 2005, 95:127-132.

15. Vitte $C$, Bennetzen $\mathrm{JL}$ : Analysis of retrotransposon structural diversity uncovers properties and propensities in angiosperm genome evolution. Proc Natl Acad Sci USA 2006, 103:17638-17643.

16. llic K, SanMiguel PJ, Bennetzen JL: A complex history of rearrangement in an orthologous region of the maize, sorghum, and rice genomes. Proc Natl Acad Sci USA 2003, 100:12265-12270.

17. Wang $Q$, Dooner HK: Remarkable variation in maize genome structure inferred from haplotype diversity at the bz locus. Proc Natl Acad Sci USA 2006, 103:17644-17649

18. International Rice Genome Sequencing Project: The map-based sequence of the rice genome. Nature 2005, 436:793-800.

19. Wing RA, Ammiraju JS, Luo M, Kim H, Yu Y, Kudrna D, Goicoechea JL, Wang W, Nelson W, Rao K, et al: The Oryza map alignment project: the golden path to unlocking the genetic potential of wild rice species. Plant Mol Biol 2005, 59:53-62

20. Ammiraju JS, Lu F, Sanyal A, Yu Y, Song X, Jiang N, Pontaroli AC, Rambo T, Currie J, Collura K, et al: Dynamic evolution of Oryza genomes is revealed by comparative genomic analysis of a genus-wide vertical data set. Plant Cell 2008, 20:3191-3209.

21. Lu F, Ammiraju JS, Sanyal A, Zhang S, Song R, Chen J, Li G, Sui Y, Song X, Cheng Z, et al: Comparative sequence analysis of MONOCULM1orthologous regions in 14 Oryza genomes. Proc Natl Acad Sci USA 2009, 106:2071-2076.

22. Sanyal A, Jetty AS, Lu F, Yu Y, Rambo T, Currie J, Kollura K, Kim HR, Chen J, $\mathrm{Ma}$ J, et al: Orthologous comparisons of the $\mathrm{Hd} 1$ region across genera reveal $\mathrm{Hd} 1$ gene lability within diploid Oryza species and disruptions to microsynteny in sorghum. Mol Biol Evol 2010, 27:2487-2506.

23. Ge S, Sang T, Lu BR, Hong DY: Phylogeny of rice genomes with emphasis on origins of allotetraploid species. Proc Natl Acad Sci USA 1999, 96:14400-14405.

24. Ma J, Jackson SA: Retrotransposon accumulation and satellite amplification mediated by segmental duplication facilitate centromere expansion in rice. Genome Res 2006, 16:251-259.

25. Roulin A, Chaparro C, Piegu B, Jackson S, Panaud O: Paleogenomic analysis of the short arm of chromosome 3 reveals the history of the African and Asian progenitors of cultivated rices. Genome Biol Evol 2010, 2:132-139.

26. Devos KM, Brown JK, Bennetzen JL: Genome size reduction through illegitimate recombination counteracts genome expansion in Arabidopsis. Genome Res 2002, 12:1075-1079.

27. McCarthy EM, Liu J, Lizhi G, McDonald JF: Long terminal repeat retrotransposons of Oryza sativa. Genome Biol 2002, 3, RESEARCH0053.

28. Tian Z, Rizzon C, Du J, Zhu L, Bennetzen JL, Jackson SA, Gaut BS, Ma J: Do genetic recombination and gene density shape the pattern of DNA elimination in rice long terminal repeat retrotransposons? Genome Res 2009, 19:2221-2230.

29. Alexander RP, Fang G, Rozowsky J, Snyder M, Gerstein MB: Annotating non-coding regions of the genome. Nat Rev Genet 2010, 11:559-571.

30. Kashkush K, Feldman M, Levy AA: Gene loss, silencing and activation in a newly synthesized wheat allotetraploid. Genetics 2002, 160:1651-1659.

31. Ginzburg LR, Bingham PM, Yoo S: On the theory of speciation induced by transposable elements. Genetics 1984, 107:331-341.

32. He L, Dooner HK: Haplotype structure strongly affects recombination in a maize genetic interval polymorphic for Helitron and retrotransposon insertions. Proc Natl Acad Sci USA 2009, 106:8410-8416.

33. Garris AJ, Tai TH, Coburn J, Kresovich S, McCouch S: Genetic structure and diversity in Oryza sativa L. Genetics 2005, 169:1631-1638.

34. Semon M, Nielsen R, Jones MP, McCouch SR: The population structure of African cultivated rice Oryza glaberrima (Steud.): evidence for elevated levels of linkage disequilibrium caused by admixture with $O$. sativa and ecological adaptation. Genetics 2005, 169:1639-1647.

35. Zhao K, Wright M, Kimball J, Eizenga G, McClung A, Kovach M, Tyagi W, Ali ML, Tung CW, Reynolds A, et al: Genomic diversity and introgression in $O$. sativa reveal the impact of domestication and breeding on the rice genome. PLoS One 2010, 5:e10780.

36. Gonzalez J, Macpherson JM, Petrov DA: A recent adaptive transposable element insertion near highly conserved developmental loci in Drosophila melanogaster. Mol Biol Evol 2009, 26:1949-1961.

37. Kovach MJ, Calingacion MN, Fitzgerald MA, McCouch SR: The origin and evolution of fragrance in rice (Oryza sativa L.). Proc Natl Acad Sci USA 2009, 106:14444-14449.

38. Caicedo AL, Williamson SH, Hernandez RD, Boyko A, Fledel-Alon A, York TL, Polato NR, Olsen KM, Nielsen R, McCouch SR, et al: Genome-wide patterns of nucleotide polymorphism in domesticated rice. PLoS Genet 2007, 3:1745-1756.

39. Kovach MJ, MCCouch SR: Leveraging natural diversity: back through the bottleneck. Curr Opin Plant Biol 2008, 11:193-200.

40. Vaughan DA, Lu B-R, Tomooka N: The evolving story of rice evolution. Plant Science 2008, 174:394-408.

41. Dubcovsky J, Ramakrishna W, SanMiguel PJ, Busso CS, Yan L, Shiloff BA, Bennetzen JL: Comparative sequence analysis of colinear barley and rice bacterial artificial chromosomes. Plant Physiol 2001, 125:1342-1353.

42. Tatusova TA, Madden TL: BLAST 2 Sequences, a new tool for comparing protein and nucleotide sequences. FEMS Microbiol Lett 1999, 174:247-250.

43. Sonnhammer EL, Durbin R: A dot-matrix program with dynamic threshold control suited for genomic DNA and protein sequence analysis. Gene 1995, 167:GC1-10.

44. Ouyang S, Zhu W, Hamilton J, Lin H, Campbell M, Childs K, ThibaudNissen F, Malek RL, Lee Y, Zheng L, et al: The TIGR Rice Genome 
Annotation Resource: improvements and new features. Nucleic Acids Res 2007, 35:D883-887.

45. Jiang N, Bao Z, Zhang X, Eddy SR, Wessler SR: Pack-MULE transposable elements mediate gene evolution in plants. Nature 2004, 431:569-573.

46. Yang L, Bennetzen JL: Distribution, diversity, evolution, and survival of Helitrons in the maize genome. Proc Natl Acad Sci USA 2009, 106:19922-19927.

47. Thompson JD, Gibson TJ, Plewniak F, Jeanmougin F, Higgins DG: The CLUSTAL_X windows interface: flexible strategies for multiple sequence alignment aided by quality analysis tools. Nucleic Acids Res 1997, 25:4876-4882

48. Tamura K, Dudley J, Nei M, Kumar S: MEGA4: Molecular Evolutionary Genetics Analysis (MEGA) software version 4.0. Mol Biol Evol 2007, 24:1596-1599.

doi:10.1186/1471-2164-12-142

Cite this article as: Tian et al: Exceptional lability of a genomic complex in rice and its close relatives revealed by interspecific and intraspecific comparison and population analysis. BMC Genomics 2011 12:142.

\section{Submit your next manuscript to BioMed Central} and take full advantage of:

- Convenient online submission

- Thorough peer review

- No space constraints or color figure charges

- Immediate publication on acceptance

- Inclusion in PubMed, CAS, Scopus and Google Scholar

- Research which is freely available for redistribution

Submit your manuscript at www.biomedcentral.com/submit 